\title{
On Chattering-Free Dynamic Sliding Mode Controller Design
}

\author{
Jeang-Lin Chang \\ Department of Electrical Engineering, Oriental Institute of Technology, Banchiao, New Taipei City 220, Taiwan \\ Correspondence should be addressed to Jeang-Lin Chang, jlchang@ee.oit.edu.tw
}

Received 7 June 2012; Revised 7 August 2012; Accepted 8 August 2012

Academic Editor: Zhiyong Chen

Copyright () 2012 Jeang-Lin Chang. This is an open access article distributed under the Creative Commons Attribution License, which permits unrestricted use, distribution, and reproduction in any medium, provided the original work is properly cited.

\begin{abstract}
For a class of linear MIMO uncertain systems, a dynamic sliding mode control algorithm that avoids the chattering problem is proposed in this paper. Without using any differentiator, we develop a modified asymptotically stable second-order sliding mode control law in which the proposed controller can guarantee the finite time convergence to the sliding mode and can show that the system states asymptotically approach to zero. Finally, a numerical example is explained for demonstrating the applicability of the proposed scheme.
\end{abstract}

\section{Introduction}

Sliding mode control (SMC) has been successfully used in controlling many uncertain systems $[1,2]$. For a system with the matched disturbance, SMC can obtain the perfect disturbance rejection during the sliding mode. The control objectives are attained by constraining the system dynamics on a properly chosen sliding variable by means of discontinuous control laws. In theory, SMC offers robust stability to systems through high-gain control with the infinite fast switching action. However, high-gain control designs suffer from the drawback of peaking phenomenon, in which the control input peaks to an extremely large value during the transient stage. The peaking phenomenon can easily violate the control saturation constraint. The SMC scheme is often discontinuous and the feedback control input needs to switch with infinite switching frequency. The discontinuous high speed switching action results in the chattering problem due to the inherent delay and other problems $[1,2]$. The chattering action may excite the unmodeled high order dynamics, which probably leads to unforeseen instability [15]. There are two major approaches reported to cope with the chattering problem. The first approach is to insert a fixed or variable boundary layer near the sliding variable [6], so that a continuous control replaces the discontinuous one when the system is inside the boundary layer. Another approach to eliminate the chattering is carried out by adding an auxiliary control input into the system such as using of fuzzy control [7, 8] and adaptive fuzzy control [9]. These mentioned methods [1-5] can give a chattering-free system, but a finite steady-state error may exist. Hence, the property of perfect disturbance rejection cannot be guaranteed.

Dynamic sliding mode control [10-14] where an integrator is used in front of the system is a special approach to eliminate the control chattering. The time derivative of the control input is treated as the new control variable for the augmented system in which the augmented system is includes the original system and the integrator. Since no boundary layer is used in the controller, the advantages of a dynamic sliding mode controller are that chattering reduction is obtained by using an integrator and the property of perfect disturbance rejection is guaranteed. Hence, dynamic sliding mode control not only removes some of the fundamental limitations of the traditional approach but also provides improved tracking accuracy under sliding mode. The main problem in implementation of dynamic sliding mode controller is the increasing information demand in which the knowledge of the derivative of the sliding variable is required. Bartolini et al. [11, 12] presented a suboptimal version of the twisting algorithm $[15,16]$ to cope with the chattering problem. However, this method requires at least the knowledge of the sign of the derivative of the sliding variable. Chen et al. [14] applied an LTR observer to overcome the problem of sliding variable estimation. Recently, Levant [15-17] presented the twisting algorithm to stabilize second-order nonlinear systems but required the knowledge 
of the derivative of the sliding variable. The super twisting algorithm $[15,16]$ does not require the output derivative to be measured but it has been originally developed and analyzed for system with relative degree one. Levant [17] proposed an exact finite time convergence differentiator which can successfully estimate the derivative of the sliding variable but required a priori bounded trajectory.

For a linear MIMO system with the matched disturbance, an alternative dynamic sliding mode controller for avoiding the chattering problem is established in this paper. We first present a modified second-order sliding mode control method to stabilize the perturbed system. Introducing a proportional-integral term of the sliding variable and an integral sign function term into the control law, the resulting control forces are chattering-free. Based on the developed second-order sliding mode technique, it is shown that the finite time convergence to the sliding mode is provided theoretically and the zero steady-state error can be guaranteed by applying the proposed control law. As a result, the control accuracy is better than those performed by the conventional boundary layer control [3-5]. Moreover, a sufficient condition for the closed-loop stability is given and the implementation of the proposed control algorithm is simple. Comparing with the conventional dynamic sliding mode controllers [10-14], the proposed method does not need any observer structure to estimate the derivative of the sliding variable. Finally, the feasibility of the proposed method is illustrated by a numerical example.

The work of this paper is organized as follows. Section 2 describes a class of uncertain MIMO linear systems and gives the problem formulation. Section 3 presents the modified second-order sliding mode method and develops the dynamic sliding mode controller design. The simulation result is included in Section 4. Section 5 offers a brief conclusion.

\section{Problem Formulation}

Consider an uncertain system satisfying the matched condition of the form

$$
\dot{\mathbf{x}}(t)=\mathbf{A} \mathbf{x}(t)+\mathbf{B}(\mathbf{u}(t)+\mathbf{d}(t, \mathbf{x})),
$$

where $\mathbf{x} \in \mathbb{R}^{n}$ is the state vector, $\mathbf{u} \in \mathbb{R}^{m}$ is the control input vector, $\mathbf{y} \in \mathbb{R}^{p}$ is the output vector, and $\mathbf{d} \in \mathbb{R}^{m}$ is the unknown matched disturbance vector with the known upper bounds $\|\mathbf{d}(t, \mathbf{x})\| \leq a_{1}$ and $\|\dot{\mathbf{d}}(t, \mathbf{x})\| \leq a_{2}$. Suppose that the system states are accessible for measurement and the pair $(\mathbf{A}, \mathbf{B})$ is stabilizable. Let the sliding variable be chosen as

$$
\mathbf{s}(t)=\mathbf{G} \mathbf{x}(t),
$$

where the matrix $\mathbf{G} \in \mathbb{R}^{m \times n}$ is designed to stabilize the reduced-order system. To satisfy the reaching and sliding condition, the control input for the conventional sliding mode controller is designed as

$$
\mathbf{u}(t)=-(\mathbf{G B})^{-1}(\mathbf{G A x}(t)+\gamma \operatorname{sign}(\mathbf{s}(t))),
$$

where $\gamma>0$ is a high gain to design such that the system reaches and slides on the sliding variable in finite time. However, a phenomenon called chattering is generated due to the discontinuous function $\operatorname{sign}(\mathbf{s}(t))$. It can be considered as the undesired chattering effect produced by the high switching action of the control input. As a result, the chattering becomes the main implementation problem of SMC. Numerous techniques have been proposed to eliminate this phenomenon in SMC [3-14]. The one of the most common solution to reduce the chattering is the boundary layer techniques [3-5]. However, the boundary layer thickness has the trade-off relation between the control performance and the chattering migration. Another drawback with applying the boundary layer methods [3-5] is the reduction of the control accuracy.

For a linear MIMO system with the matched disturbance, in this paper we propose a dynamic sliding mode control algorithm in which the proposed procedure can effectively reduce the chattering effect. We first develop a modified second-order sliding mode control method to stabilize the perturbed systems. Introducing a proportional-integral term of the sliding variable and an integral sign function term into the controller, the finite time convergence to the sliding mode is guaranteed by applying the developed second-order sliding mode technique. Moreover, all the states of the system asymptotically approach to zero once the system is in the sliding mode.

\section{Dynamic Sliding Mode Controller Design}

In this section, we propose a dynamic sliding mode control algorithm which can successfully avoid the chattering in the linear MIMO system with the matched disturbance. A modified second-order sliding mode control algorithm that does not require the derivative of the sliding variable is presented. The proposed control law can guarantee the finite time convergence to the sliding mode and stabilize the reduced-order system in which the system states asymptotically approach to zero.

For the sliding variable (2), we design the dynamic sliding mode controller as

$$
\begin{gathered}
\mathbf{u}(t)=-(\mathbf{G B})^{-1}\left(\mathbf{G A x}(t)+\mathbf{L}_{1} \mathbf{s}(t)+\mathbf{L}_{2} \int_{0}^{t} \mathbf{s}(\tau) d \tau\right. \\
\left.+\mathbf{K} \int_{0}^{t} \operatorname{sign}(\mathbf{s}(\tau)) d \tau\right),
\end{gathered}
$$

where $\mathbf{L}_{1} \in \mathbb{R}^{m \times m}, \mathbf{L}_{2} \in \mathbb{R}^{m \times m}$, and $\mathbf{K} \in \mathbb{R}^{m \times m}$ are the positive definite diagonal matrix given by

$$
\begin{gathered}
\mathbf{L}_{1}=\operatorname{diag}\left(l_{11}, \ldots, l_{1 m}\right), \quad \mathbf{L}_{2}=\operatorname{diag}\left(l_{21}, \ldots, l_{2 m}\right), \\
\mathbf{K}=\operatorname{diag}\left(k_{1}, \ldots, k_{m}\right) .
\end{gathered}
$$


Taking the time derivative of (2) and substituting the control input (4) into it can obtain

$$
\begin{aligned}
\dot{\mathbf{s}}(t)= & \mathbf{G} \dot{\mathbf{x}}(t)=\mathbf{G A x}(t)+\mathbf{G B}(\mathbf{u}(t)+\mathbf{d}(t, \mathbf{x})) \\
= & -\mathbf{L}_{1} \mathbf{s}(t)-\mathbf{L}_{2} \int_{0}^{t} \mathbf{s}(\tau) d \tau-\mathbf{K} \int_{0}^{t} \operatorname{sign}(\mathbf{s}(\tau)) d \tau \\
& +\mathbf{G B d}(t, \mathbf{x}) .
\end{aligned}
$$

Further differentiating (6) with time yields the following dynamics:

$$
\ddot{\mathbf{s}}(t)+\mathbf{L}_{1} \dot{\mathbf{s}}(t)+\mathbf{L}_{2} \mathbf{s}(t)=-\mathbf{K} \operatorname{sign}(\mathbf{s}(t))+\mathbf{f}(t),
$$

where $\mathbf{f}(t)=\mathbf{G B} \dot{\mathbf{d}}(\mathbf{x}, t)=\left[f_{1} \cdots f_{m}\right]^{T} \in \mathbb{R}^{m}$. According to the linear algebraic theory, we have $\|\mathbf{f}(t)\|=\|\mathbf{G B \dot { d }}(\mathbf{x}, t)\| \leq$ $a_{2}\|\mathbf{G B}\|$ and $\left|f_{i}(t)\right| \leq\|\mathbf{f}(t)\|$ for $1 \leq i \leq m$.

Lemma 1. Consider the unperturbed system as

$$
\ddot{\sigma}(t)+l_{1} \dot{\sigma}(t)+l_{2} \sigma(t)=-k \operatorname{sign}(\sigma(t)),
$$

where the gains $l_{1}, l_{2}$, and $k$ are positive constants. If the parameters $l_{1}>0$ and $l_{2}>0$ are chosen to satisfy the condition $l_{2}<l_{1}^{2} / 4$, then $\sigma(t)$ and $\dot{\sigma}(t)$ asymptotically converge to zero in finite time for a sufficiently large value of $k$.

Proof. Since the condition $l_{2}<l_{1}^{2} / 4$ holds, the roots of the characteristic equation, $s^{2}+l_{1} s+l_{2}=0$, are real, distinct, and negative. Assume now for simplicity that the initial conditions are $\sigma\left(t_{0}\right)=0$ and $\dot{\sigma}\left(t_{0}\right)>0$. Thus the trajectory enters the half-plane $\sigma(t)>0$ (quadrant I), as shown in Figure 1. When $\sigma(t)>0$, we from (8) obtain $\ddot{\sigma}(t)+l_{1} \dot{\sigma}(t)+$ $l_{2} \sigma(t)=-k$ and know its equivalent point as $(\sigma, \dot{\sigma})=$ $\left(-k / l_{2}, 0\right)$. Let the function $g(t)$ be generated by

$$
g(t)=\dot{\sigma}(t)+r \sigma(t)+\frac{k}{\mu} \operatorname{sign}(\sigma(t))
$$

where $\mu+r=l_{1}$ and $\mu r=l_{2}$. The parameters $\mu>0$ and $r>0$ are real constants. Since $\sigma(t)>0$, it follows from $\ddot{\sigma}(t)+$ $l_{1} \dot{\sigma}(t)+l_{2} \sigma(t)+k=0$ that

$$
\begin{aligned}
\frac{d g(t)}{d t}+\mu g(t)= & \frac{d}{d t}\left(\dot{\sigma}(t)+r \sigma(t)+\frac{k}{\mu}\right) \\
& +\mu\left(\dot{\sigma}(t)+r \sigma(t)+\frac{k}{\mu}\right) \\
= & \ddot{\sigma}(t)+(\mu+r) \dot{\sigma}(t)+\mu r \sigma(t)+k \\
= & \ddot{\sigma}(t)+l_{1} \dot{\sigma}(t)+l_{2} \sigma(t)+k=0 .
\end{aligned}
$$

Hence, we can obtain $g(t)=g(0) e^{-\mu t}$. It follows that the upper bound of $g(t)$ is $|g(t)| \leq C e^{-\mu t}$ where $C>0$ is a constant. Choose a Lyapunov function as $V(t)=(1 / 2) \sigma^{2}(t)$ and then obtain its time derivative as

$$
\begin{aligned}
\dot{V}(t) & =\sigma(t) \dot{\sigma}(t)=\sigma(t)\left(g(t)-\frac{k}{\mu} \operatorname{sign}(\sigma(t))-r \sigma(t)\right) \\
& \leq C|\sigma(t)| e^{-\mu t}-\frac{k}{\mu}|\sigma(t)|-r \sigma^{2}(t) .
\end{aligned}
$$

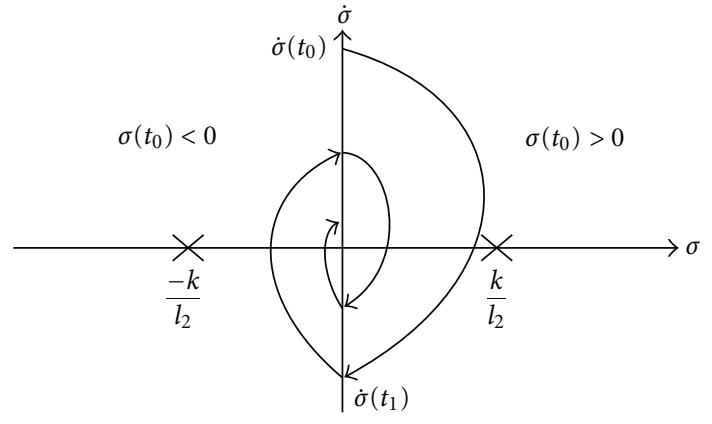

FIGURE 1: Phase paths of the modified second-order sliding mode system.

Since $\mu>0$, there exists a finite time, $T_{1}>0$, such that $C e^{-\mu t}<(k / \mu)-\rho$ for a sufficiently large gain $k$ and $t>T_{1}$, where $\rho>0$ is a constant. Hence,

$$
\dot{V}(t) \leq-\rho|\sigma(t)|-r \sigma^{2}(t) \leq-\rho|\sigma(t)| \text { for } t>T_{1} .
$$

The above equation implies that the function $\sigma(t)$ converges to zero in finite time. Let the trajectory of (8) intersect next time with the axis $\sigma(t)=0$ at the point $\dot{\sigma}\left(t_{1}\right)$. Since the roots of the characteristic equation, $s^{2}+l_{1} s+l_{2}=0$, are stable, we know that the spiral trajectories converge to the equivalent point and the behavior of $\dot{\sigma}(t)$ changes monotonously. Hence,

$$
\frac{\left|\dot{\sigma}\left(t_{1}\right)\right|}{\left|\dot{\sigma}\left(t_{0}\right)\right|}=q<1 .
$$

Extending the trajectory into the half plane $\sigma(t)<0$ after a similar reasoning achieves that the successive crossing the axis $\sigma(t)=0$ satisfies the inequality $\left|\dot{\sigma}\left(t_{i+1}\right)\right| /\left|\dot{\sigma}\left(t_{i}\right)\right|=q<1$. Therefore, its solutions cross the axis $\sigma(t)=0$ from quadrant II to quadrant I, and from quadrant IV to quadrant III. Every trajectory of the system crosses the axis $\sigma(t)=0$ in finite time. After gluing these paths along the line $\sigma(t)=0$, we obtain the phase portrait of the system, as shown in Figure 1. This algorithm features a twisting of the phase portrait around the origin and an infinite number encircling the origin occurs. According to Levant's papers [14-16], the total convergence time is estimated as

$$
T \leq \sum\left|\dot{\sigma}\left(t_{i}\right)\right| \leq\left|\dot{\sigma}\left(t_{0}\right)\right|\left(1+q+q^{2}+\cdots\right)=\frac{\left|\dot{\sigma}\left(t_{0}\right)\right|}{1-q} .
$$

As a result, we can show that the trajectories perform rotations around the origin while converging in finite time to the origin of the phase plane. The finite time convergence to the origin is due to switching between two different control amplitudes as the trajectory comes nearer to the origin [18]. The proof of the theorem is finished.

Lemma 2. Consider the following system:

$$
\ddot{\sigma}(t)+l_{1} \dot{\sigma}(t)+l_{2} \sigma(t)=-k \operatorname{sign}(\sigma(t))+f(t),
$$


where the function $f(t)$ has the upper bound $|f(t)| \leq \eta$ and $\eta>0$ is a known constant. If the parameters $l_{1}>0$ and $l_{2}>0$, and the gain $k$ are chosen to satisfy the following conditions:

$$
l_{2}<\frac{l_{1}^{2}}{4}, \quad k>\eta
$$

then $\sigma(t)$ and $\dot{\sigma}(t)$ converge to zero in finite time.

Proof. We take the parameters $l_{1}$ and $l_{2}$ to satisfy the condition $l_{2}<l_{1}^{2} / 4$ and then obtain the characteristic polynomial of system (15) having two distinct real roots $\lambda_{1,2}=-\alpha,-\beta$, where $\alpha>0, \beta>0, \beta>\alpha, l_{1}=\alpha+\beta$, and $l_{2}=\alpha \beta$. When the condition $\sigma(t)>0$ holds, (15) becomes

$$
\ddot{\sigma}(t)+l_{1} \dot{\sigma}(t)+l_{2} \sigma(t)=-k+f(t) .
$$

Let $v_{1}=\sigma+\left(k / l_{2}\right)$ and $v_{2}=\dot{v}_{1}=\dot{\sigma}$. It follows that

$$
\dot{\mathbf{v}}(t)=\left[\begin{array}{cc}
0 & 1 \\
-l_{2} & -l_{1}
\end{array}\right]\left[\begin{array}{l}
v_{1}(t) \\
v_{2}(t)
\end{array}\right]+\left[\begin{array}{c}
0 \\
f(t)
\end{array}\right]=\boldsymbol{\Phi v}(t)+\mathbf{b} f(t),
$$

where

$$
\mathbf{v}=\left[\begin{array}{ll}
v_{1}^{T} & v_{2}^{T}
\end{array}\right]^{T} \in \mathbb{R}^{2}, \quad \boldsymbol{\Phi}=\left[\begin{array}{cc}
0 & 1 \\
-l_{2} & -l_{1}
\end{array}\right], \quad \mathbf{b}=\left[\begin{array}{l}
0 \\
1
\end{array}\right] .
$$

Write the above dynamic equation as its solution in an explicit form

$$
\begin{aligned}
\mathbf{v}(t) & =\mathbf{e}^{\Phi t} \mathbf{v}(0)+\mathbf{e}^{\Phi t} \int_{0}^{t} \mathbf{e}^{\Phi \tau} \mathbf{b} f(\tau) d \tau \\
& =\mathbf{e}^{\Phi t} \mathbf{v}(0)+\int_{0}^{t} \mathbf{e}^{\Phi \tau} \mathbf{b} f(t-\tau) d \tau,
\end{aligned}
$$

where

$$
\mathbf{e}^{\Phi t}=\left[\begin{array}{cc}
\frac{1}{\beta-\alpha}\left(\beta e^{-\alpha t}-\alpha e^{-\beta t}\right) & \frac{1}{\beta-\alpha}\left(e^{-\alpha t}-e^{-\beta t}\right) \\
\frac{\alpha \beta}{\alpha-\beta}\left(e^{-\alpha t}-e^{-\beta t}\right) & \frac{1}{\alpha-\beta}\left(\alpha e^{-\alpha t}-\beta e^{-\beta t}\right)
\end{array}\right] .
$$

The upper bounds of $v_{1}(t)$ and $v_{2}(t)$ can be constructed as

$$
\begin{aligned}
\left|v_{1}(t)\right| & \leq C_{1} e^{-\alpha t}+\frac{1}{\beta-\alpha} \int_{0}^{t}\left|e^{-\alpha \tau}-e^{-\beta \tau}\right||f(t-\tau)| d \tau \\
& \leq C_{1} e^{-\alpha t}+\frac{\eta}{\beta-\alpha} \int_{0}^{t}\left|e^{-\alpha \tau}-e^{-\beta \tau}\right| d \tau \\
& =C_{1} e^{-\alpha t}+\frac{\eta}{\beta-\alpha} \int_{0}^{\infty}\left(e^{-\alpha \tau}-e^{-\beta \tau}\right) d \tau \\
& =C_{1} e^{-\alpha t}+\frac{\eta}{\beta-\alpha}\left(\frac{\beta-\alpha}{\alpha \beta}\right)=C_{1} e^{-\alpha t}+\frac{\eta}{l_{2}}, \\
\left|v_{2}(t)\right| & \leq C_{2} e^{-\alpha t}+\frac{\eta}{\beta-\alpha}\left(\int_{0}^{t}\left|\alpha e^{-\alpha \tau}-\beta e^{-\beta \tau}\right| d \tau\right) \\
& \leq C_{2} e^{-\alpha t}+\frac{\eta}{\beta-\alpha}
\end{aligned}
$$

$$
\begin{aligned}
& \times\left(\int_{0}^{\infty}\left|\alpha e^{-\alpha \tau}-\alpha e^{-\beta \tau}+(\alpha-\beta) e^{-\beta \tau}\right| d \tau\right) \\
= & C_{2} e^{-\alpha t}+\frac{\eta}{\beta-\alpha} \\
& \times\left(\int_{0}^{\infty}\left(\alpha e^{-\alpha \tau}-\alpha e^{-\beta \tau}\right) d \tau+(\beta-\alpha) \int_{0}^{\infty} e^{-\beta \tau} d \tau\right) \\
= & C_{2} e^{-\alpha t}+\frac{\eta}{\beta-\alpha}\left(\alpha \frac{\beta-\alpha}{\alpha \beta}+\frac{\beta-\alpha}{\beta}\right) \\
= & C_{2} e^{-\alpha t}+\frac{2 \alpha \eta}{l_{2}},
\end{aligned}
$$

where $C_{1}>0$ and $C_{2}>0$ are constants. It follows that

$$
\left|v_{1}(t)\right| \leq C_{1} e^{-\alpha t}+\frac{\eta}{l_{2}}, \quad\left|v_{2}(t)\right| \leq C_{2} e^{-\alpha t}+\frac{2 \eta \alpha}{l_{2}} .
$$

Since $v_{1}=\sigma+\left(k / l_{2}\right)$, we can from (23) obtain

$$
\left|\sigma(t)+\frac{k}{l_{2}}\right| \leq C_{1} e^{-\alpha t}+\frac{\eta}{l_{2}} .
$$

This part shows that the ball of radius $r=\eta / l_{2}$ with center located at $\left(k / l_{2}, 0\right)$ is an attractor $B_{s 1}$. Similar to the work, we have, when $\sigma(t)<0$, the ball of radius $r$, with center located at $\left(-k / l_{2}, 0\right)$ as another attractor $B_{s 2}$. Choose the gain $k$ to satisfy the inequality $k>\eta$ and then we have

$$
\left(\frac{k}{l_{2}}-r\right)=\frac{k}{l_{2}}-\frac{\eta}{l_{2}}>0, \quad\left(-\frac{k}{l_{2}}+r\right)=\frac{-k}{l_{2}}+\frac{\eta}{l_{2}}<0 .
$$

It follows from the above two inequalities that the two attractors $B_{s 1}$ and $B_{s 2}$ do not intersect each other, and the behavior of the perturbed system (15) will be qualitatively similar to the behavior of the nominal system. Therefore, the perturbed system converges to the origin in the same way of the nominal system and the condition that $\sigma(t)$ and $\dot{\sigma}(t)$ converge to zero in finite time can be guaranteed. We finish the proof of the lemma.

Theorem 3. Consider system (1) with the sliding variable (2) and the control input (4). Let $\eta=a_{2}\|\mathbf{G B}\|$. If the elements of these matrices $\mathbf{L}_{1}, \mathbf{L}_{2}$, and $\mathbf{K}$ satisfy the following conditions:

$$
l_{2 i}<\frac{l_{1 i}^{2}}{4}, \quad k_{i}>\eta, \quad \text { for } i=1, \ldots, m,
$$

then one can show that the system states asymptotically approach to zero.

Proof. We first express system (7) as a set of second-order systems with the form

$$
\begin{array}{r}
\ddot{s}_{i}(t)+l_{1 i} \dot{s}(t)+l_{2 i} s(t)=-k_{i} \operatorname{sign}\left(s_{i}(t)\right)+f_{i}(t) \\
\text { for } i=1, \ldots, m,
\end{array}
$$




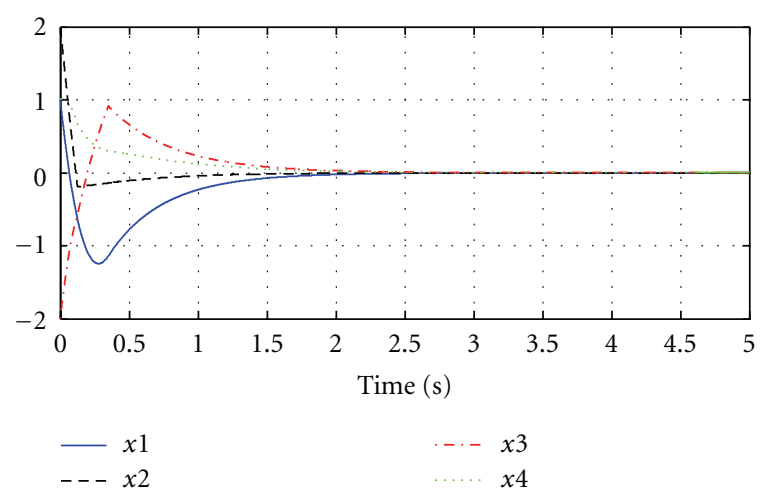

FIGURE 2: System states with applying the sign function.

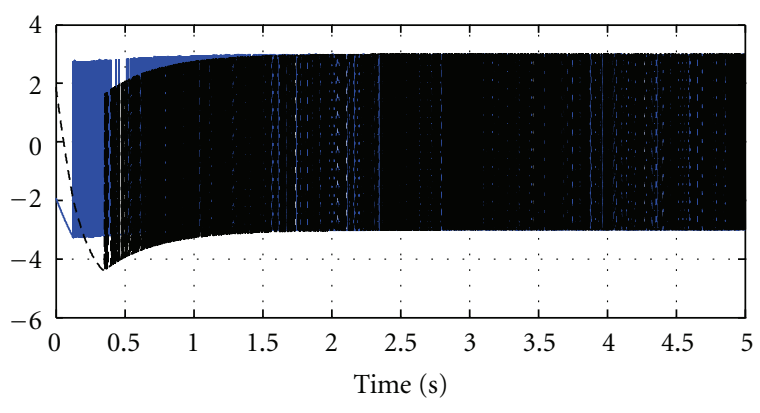

$-u 1$

$---u 2$

Figure 3: Control inputs with applying the sign function.

where $\left|f_{i}(t)\right| \leq \eta$ for $i=1, \ldots, m$ where $\eta=a_{2}\|\mathbf{G B}\|>0$ is a known constant. Applying the result of Lemma 2 into (27), if the parameters $l_{1 i}, l_{i 2}$, and $k_{i}$ can satisfy

$$
l_{2 i}<\frac{l_{1 i}^{2}}{4}, \quad k_{i}>\eta, \quad \text { for } i=1, \ldots, m,
$$

then $s_{i}(t)$ and $\dot{s}_{i}(t)$ asymptotically converge to zero in finite time according to Lemma 2. When the condition $\mathbf{s}(t)=\mathbf{0}$ is guaranteed, it follows from the concept of equivalent control $[1,2]$ that the system dynamics in the sliding mode is

$$
\dot{\mathbf{x}}(t)=\left(\mathbf{A}-\mathbf{B}(\mathbf{G B})^{-1} \mathbf{G A}\right) \mathbf{x}(t) .
$$

Since the nonzero eigenvalues of the matrix $\mathbf{A}-\mathbf{B}(\mathbf{G B})^{-1} \mathbf{G A}$ are placed in the left-half plane, we can conclude that the system states asymptotically approach to zero and finally finish the proof of the theorem.

\section{Numerical Example}

To demonstrate the effectiveness of the proposed method, we consider an unstable batch reactor where the matched

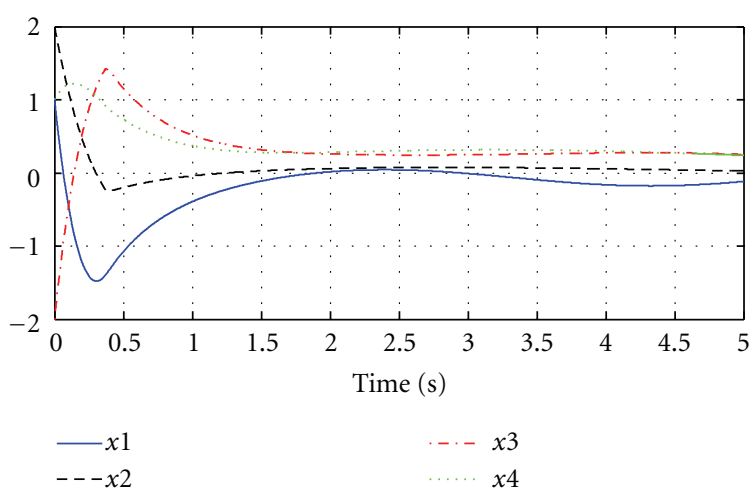

FIGURE 4: System states with applying the saturation function.

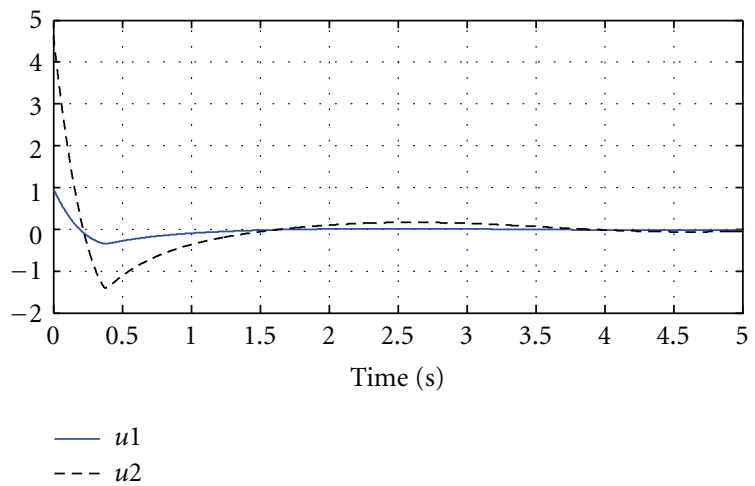

FIGURE 5: Control inputs with applying the saturation function.

disturbance is introduced into the system and its state space form can be given by

$$
\begin{aligned}
\dot{\mathbf{x}}(t)= & {\left[\begin{array}{cccc}
1.38 & -0.2077 & 6.715 & -5.676 \\
-0.5814 & -4.29 & 0 & 0.675 \\
1.067 & 4.273 & -6.654 & 5.893 \\
0.048 & 4.273 & 1.343 & -2.104
\end{array}\right] \mathbf{x}(t) } \\
& +\left[\begin{array}{cc}
0 & 0 \\
5.679 & 0 \\
1.136 & -3.146 \\
1.136 & 0
\end{array}\right]\left(\mathbf{u}(t)+\left[\begin{array}{c}
1.5 \sin (0.5 t) \\
1 \cos \left(\frac{\pi t}{2}\right)
\end{array}\right]\right) .
\end{aligned}
$$

The sliding variable is chosen as

$$
\mathbf{s}(t)=\left[\begin{array}{cccc}
-0.0106 & 0.1684 & 0 & 0.0383 \\
-0.1931 & 0.0155 & -0.3179 & 0.2404
\end{array}\right] \mathbf{x}(t)
$$

where the nonzero eigenvalues of the system in the sliding mode are assigned as $\{-2,-3\}$. We design the conventional 


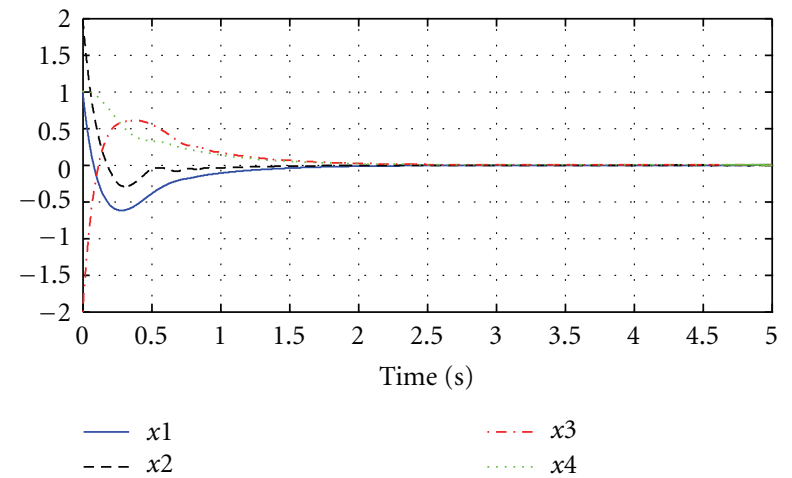

FIGURE 6: System states with applying the proposed method.

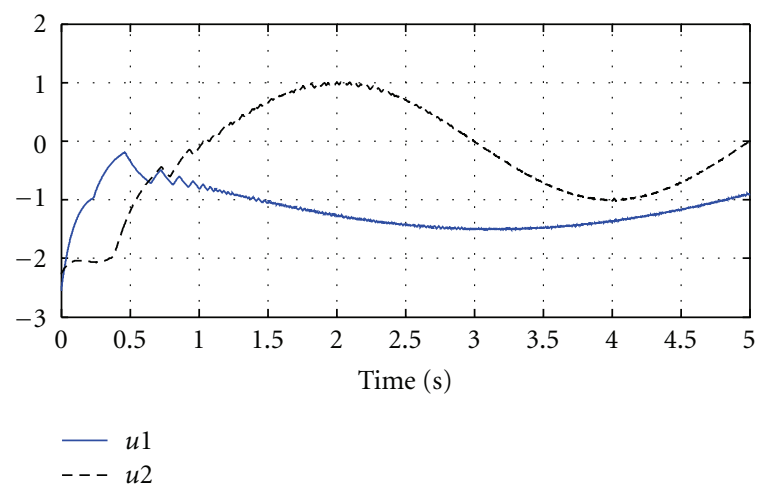

FIGURE 7: Control inputs with applying the proposed method.

sliding mode controller and the boundary layer controller, respectively, as

$$
\begin{aligned}
\mathbf{u}(t)= & -\left[\begin{array}{cccc}
-0.1108 & -0.5565 & -0.02 & 0.0934 \\
-0.6031 & -0.3576 & 1.1415 & -1.2726
\end{array}\right] \mathbf{x}(t) \\
& -3 \operatorname{sign}(\mathbf{s}(t)) \\
\mathbf{u}(t)= & -\left[\begin{array}{lllc}
-0.1108 & -0.5565 & -0.02 & 0.0934 \\
-0.6031 & -0.3576 & 1.1415 & -1.2726
\end{array}\right] \mathbf{x}(t) \\
& -3 \operatorname{sat}(\mathbf{s}(t), 0.05)
\end{aligned}
$$

The control input with applying the proposed algorithm is given by

$$
\begin{aligned}
\mathbf{u}(t)= & -\left[\begin{array}{llll}
-0.1108 & -0.5565 & -0.02 & 0.0934 \\
-0.6031 & -0.3576 & 1.1415 & -1.2726
\end{array}\right] \mathbf{x}(t) \\
& -10 \mathbf{s}(t)-2 \int_{0}^{t} \mathbf{s}(\tau) d \tau-3 \int_{0}^{t} \operatorname{sign}(\mathbf{s}(\tau)) d \tau .
\end{aligned}
$$

Three cases are simultaneously simulated under the initial condition $\mathbf{x}(0)=\left[\begin{array}{llll}1 & 2 & -2 & 1\end{array}\right]^{T}$ and the simulation is carried out at a fixed step size of 1 milliseconds. The time responses of the system states in the three cases are shown in Figures 2, 4, and 6, respectively. As can be seen from these figures, the proposed method obtains the zero steady-state error. Figures 3, 5, and 7 show the control inputs in the three

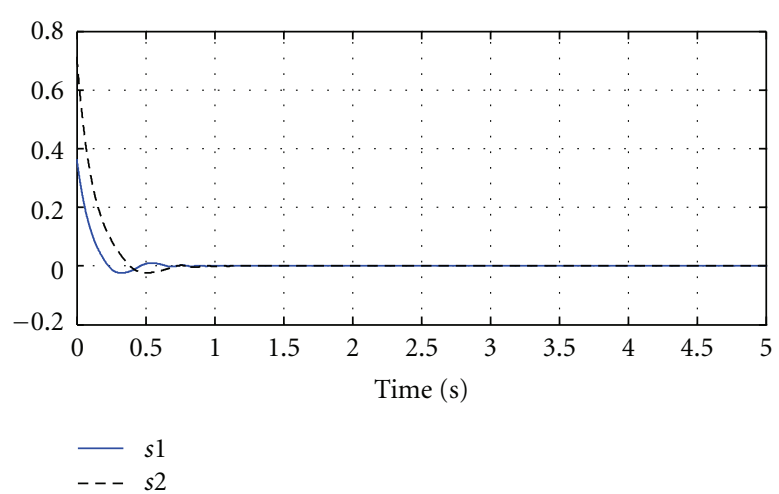

FIGURE 8: Sliding variables with applying the proposed method.

cases, respectively. As illustrated, our control force does not exhibit any chattering effects. The responses of the sliding variable with applying the proposed method are given in Figure 8. It is clear from the figure that our control law can guarantee the finite time convergence to the sliding mode. As a result, the simulation results verify the good performance of the proposed strategy.

\section{Conclusions}

In this paper we have proposed a modified second-order sliding mode control algorithm to avoid the chattering problem for a MIMO uncertain system. The developed approach does not require the derivative of the sliding variable, thus eliminating the requirement of designing a differentiator. Under the proposed dynamic sliding mode controller, we show that the finite time convergence to the sliding mode is guaranteed and the system states can asymptotically approach to zero. Simulation results demonstrate that the proposed control scheme exhibits reasonably good system performance.

\section{References}

[1] C. Edwards and S. K. Spurgeon, Sliding Mode Control Theory and Application, Taylor \& Francis, London, UK, 1998.

[2] V. I. Utkin, J. Guldner, and J. Shi, Sliding Mode Control in Electro-Mechanical Systems, CRC Press, 2009.

[3] G. Bartolini, "Chattering phenomena in discontinuous control systems," International Journal of Systems Science, vol. 20, no. 12, pp. 2471-2481, 1989.

[4] J. A. Burton and A. S. I. Zinober, "Continuous approximation of variable structure control," International Journal of Systems Science, vol. 17, no. 6, pp. 875-885, 1986.

[5] J. J. Slotine and S. S. Sastry, "Tracking control of nonlinear systems using sliding surface with application to robot manipulator," International Journal of Control, vol. 38, no. 2, pp. 931-938, 1983.

[6] L. K. Wong, F. H. F. Leung, and P. K. S. Tam, "A chattering elimination algorithm for sliding mode control of uncertain non-linear systems," Mechatronics, vol. 8, no. 7, pp. 765-775, 1998. 
[7] M. Roopaei and M. Zolghadri Jahromi, "Chattering-free fuzzy sliding mode control in MIMO uncertain systems," Nonlinear Analysis, vol. 71, no. 10, pp. 4430-4437, 2009.

[8] T. Shaocheng, C. Bin, and W. Yongfu, "Fuzzy adaptive output feedback control for MIMO nonlinear systems," Fuzzy Sets and Systems, vol. 156, no. 2, pp. 285-299, 2005.

[9] N. Noroozi, M. Roopaei, and M. Z. Jahromi, "Adaptive fuzzy sliding mode control scheme for uncertain systems," Communications in Nonlinear Science and Numerical Simulation, vol. 14, no. 11, pp. 3978-3992, 2009.

[10] Sira-Ramirez, "On the dynamical sliding mode control of nonlinear systems," International Journal of Control, vol. 57, pp. 1039-1061, 1993.

[11] G. Bartolini and P. Pydynowski, "An improved, chattering free, V.S.C. scheme for uncertain dynamical systems," IEEE Transactions on Automatic Control, vol. 41, no. 8, pp. 12201226, 1996.

[12] G. Bartolini, A. Ferrara, and E. Usai, "Chattering avoidance by second-order sliding mode control," IEEE Transactions on Automatic Control, vol. 43, no. 2, pp. 241-246, 1998.

[13] G. Bartolini, A. Ferrara, E. Usai, and V. I. Utkin, "On multiinput chattering-free second-order sliding mode control," IEEE Transactions on Automatic Control, vol. 45, no. 9, pp. 1711-1717, 2000.

[14] M. S. Chen, C. H. Chen, and F. Y. Yang, "An LTR-observerbased dynamic sliding mode control for chattering reduction," Automatica, vol. 43, no. 6, pp. 1111-1116, 2007.

[15] A. Levant, "Sliding order and sliding accuracy in the sliding mode control," International Journal of Control, vol. 58, pp. 1247-1263, 1993.

[16] A. Levant, "Higher-order sliding modes, differentiation and output-feedback control," International Journal of Control, vol. 76, no. 9-10, pp. 924-941, 2003.

[17] A. Levant, "Robust exact differentiation via sliding mode technique," Automatica, vol. 34, no. 3, pp. 379-384, 1998.

[18] M. Khan, S. Spurgeon, and A. Levant, "Simple output feedback 2-sliding controller for systems of relative degree two," in Proceedings of the European Control Conference (ECC '03), Cambridge, UK, 2003. 

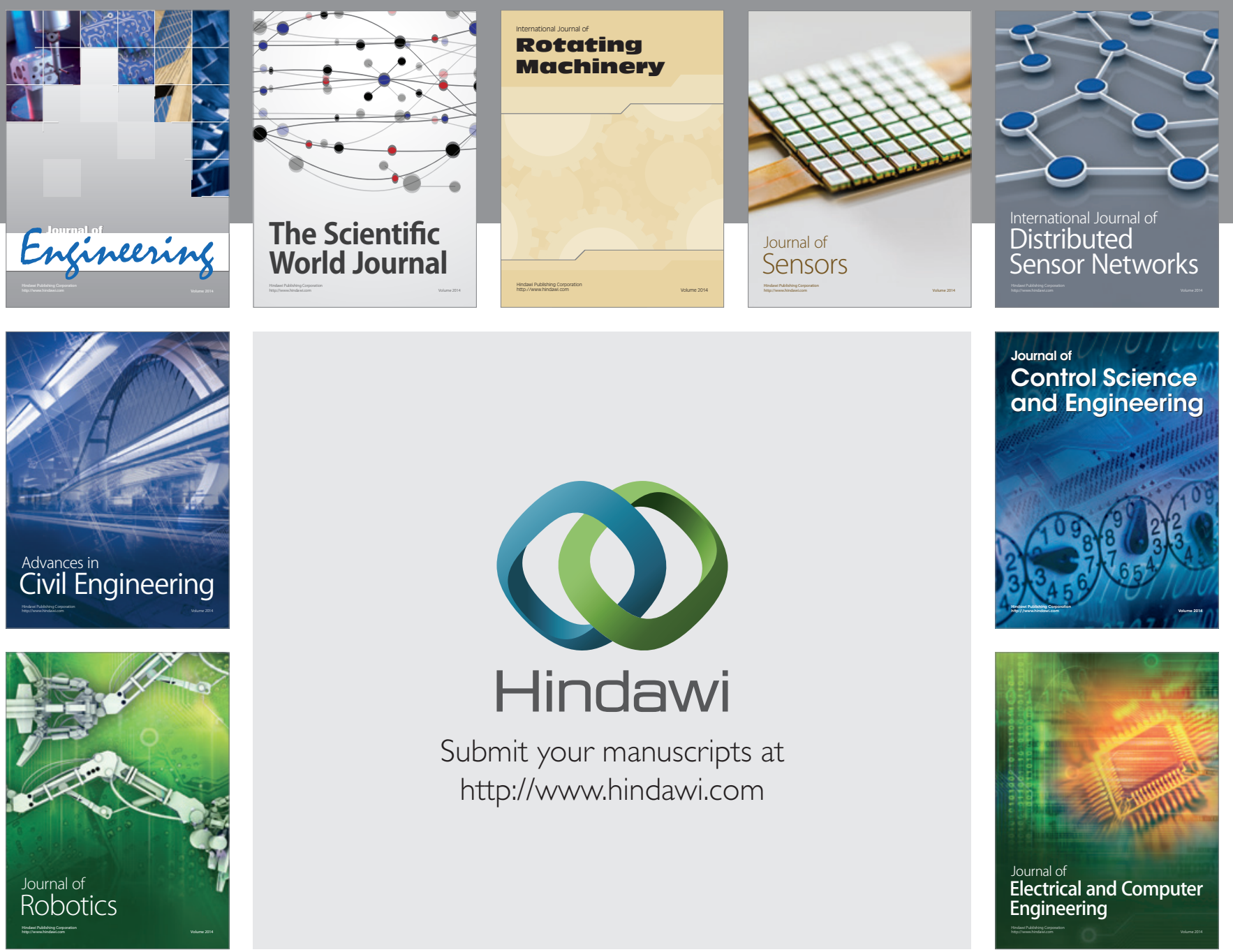

Submit your manuscripts at

http://www.hindawi.com
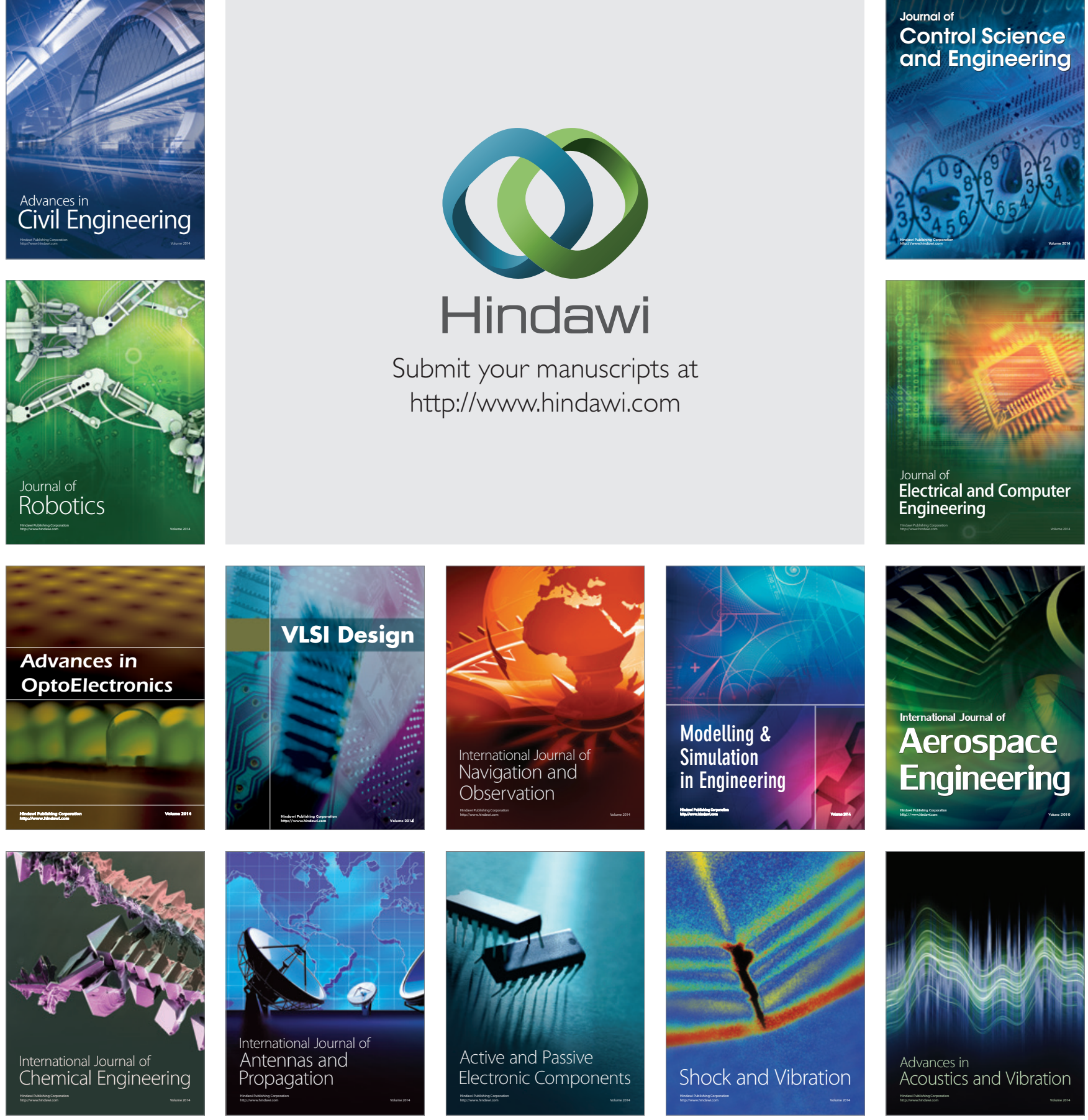